\title{
A glaucoma equity profile: correlating disease distribution with service provision and uptake in a population in Northern England, UK
}

${ }^{1}$ Public Health, NHS Yorkshire and Humber, Leeds, UK

${ }^{2}$ Community Eye Health, NHS Leeds and Leeds Teaching Hospitals NHS Trust, Leeds, UK

${ }^{3}$ Public Health, NHS Leeds, Leeds, UK

${ }^{4}$ Information Department, NHS Leeds, Leeds, UK

Correspondence: F Day, NHS Yorkshire and Humber, Blenheim House,

Duncombe Street,

Leeds LS1 4PL,

UK

Tel: +44 113295 2174;

Fax: + 441132952026

E-mail: Fiona.day@

yorksandhumber.nhs.uk

Received: 14 January 2010 Accepted in revised form: 19 April 2010

Published online: 28 May 2010

This work was presented at the Faculty of Public Health Annual Scientific Conference 2009

\begin{abstract}
Aims Glaucoma is a significant health problem, with associated inequalities. Equity profiles are an established public health tool to examine the scale of health inequalities and to imbed action into the commissioning cycle. This is the first equity profile conducted in the United Kingdom for an ophthalmic condition. This methodology also provides a model for use in other localities and for other eye conditions.

Methods Existing services were mapped and need identified. A wide variety of data sources were analysed. Mapping was undertaken using Mapinfo Professional Geographical Information Systems software. Statistical analysis was conducted using Microsoft Excel 2003.

Results No single data source provided a fully informed perspective. A clear mismatch between areas of deprivation and location of optometry was observed. Secondary analysis of electronic patient records revealed a significant association between 'late presentation' and older age (mean age of late presenters $=76.4$ years, $95 \% \mathrm{CI}=75.1-77.6$ compared with earlier presenters, 72.4 years, 95\% $\mathrm{CI}=$ 71.7-73.1). Late presentation was also associated with living in an area of high deprivation $\left(\chi^{2}=7.1,1 \mathrm{df}, P<0.05\right)$. Ethnicity data was poorly recorded. Qualitative data provided invaluable insights. Conclusions Increasing access to services involves collaboration with optometrists, ophthalmologists, public health, and
\end{abstract}

F Day', JC Buchan², A Cassells-Brown², J Fear³, R Dixon ${ }^{4}$ and F Wood ${ }^{4}$ commissioners. It is no longer acceptable to rely on private high street optometry to provide primary eye care services in areas of high need. Outreach services must be developed and evaluated in areas of relative deprivation if world class eye services are to be achieved.

Eye (2010) 24, 1478-1485; doi:10.1038/eye.2010.73; published online 28 May 2010

Keywords: glaucoma; equity; public health

\section{Introduction}

Glaucoma is a major ophthalmic problem whose management accounts for approximately $25 \%$ of general ophthalmologist follow-up attendances and $15 \%$ of new ophthalmology referrals in the United Kingdom. ${ }^{1}$ Glaucoma also constitutes a major public health problem; the World Health Organisation estimates that glaucoma is responsible for $12.3 \%$ of the 161 million blind people worldwide, and in the United Kingdom, glaucoma accounts for $10-15 \%$ of sight loss registrations. ${ }^{1,2}$

The focus of the ophthalmic community has been on patients presenting to eye departments by whatever route of referral. There is increasing awareness among ophthalmologists of the need to consider the eye health of the whole population. This is particularly pressing for glaucoma: of those with glaucoma in the United Kingdom, it is estimated that only onethird are currently detected. ${ }^{3}$ It has been shown that deprivation at both area and individual 
level is a risk factor for late presentation and resultant blindness from glaucoma. ${ }^{4}$ Glaucoma thus forms both a cause and a consequence of health inequalities in the United Kingdom.

Equity profiles are an established public health tool for embedding evidence on health inequalities into planning, commissioning, and service delivery. They quantify how fairly services or other resources are distributed in relation to the health needs of different groups and areas, and identify any remedial actions, which need to be taken. ${ }^{5}$ This is the first equity profile conducted in the United Kingdom for an ophthalmic condition, and also provides a model for others wishing to perform similar assessments in their own locality. The aim was to develop an equity profile for primary open angle glaucoma (POAG), as part of a broader equity audit to reduce associated inequalities. The objectives were as follows:

- to pilot method for use for other ophthalmic conditions as part of broader ophthalmic Public Health work in Leeds and beyond,

- to use the equity profile to make recommendations for local action including standards for future audit,

- to use findings to influence local commissioning and care pathways,

- to disseminate findings to the appropriate audience(s),

- to complete the cycle of equity audit after an appropriate period of time.

The equity profile was conducted in Leeds, a city in the north of England, with a population of 794777 in April 2008. There is a high level of relative deprivation: approximately $20 \%$ of the Leeds population resides in the $10 \%$ most deprived areas (Lower Super Output Areas, LSOAs) of England. This paper shows the methodology and, because of space restrictions, only the principle findings are presented here. A full copy of the equity profile is available from the lead author on request.

\section{Materials and methods}

The first step was to map existing services, in terms of general ophthalmic services (GOS) and hospital eye services and intermediate levels of care provision. Mapping was undertaken using Mapinfo Professional Geographical Information Systems (GIS) software, which enabled the location of services to be shown on geographical maps, which included road infrastructure, areas of deprivation, and other domains as required. The indicator of deprivation used throughout the equity profile was the Index of Multiple Deprivation 2007, which stratifies units of around 1500 residents (referred to as LSOA) according to their scores in various deprivation domains.
The second step was to identify need, in terms of the distribution of glaucoma, known risk factors for glaucoma, and relative deprivation in the city. National glaucoma prevalence estimates were used, ${ }^{6}$ and were collaborated by the Vision2020 Leeds Rapid Ophthalmic Needs Assessment (personal communication) and the National Eye Health Epidemiological Model. The estimated distribution of glaucoma cases was calculated at a local level within the city based on estimates per individual general practice, weighted for 2001 censusbased ethnicity data, and age based on January 2008 general practice registered population. This age and ethnicity data was also used to create age and ethnicity distribution maps of Leeds using GIS.

The third step was to explore and analyse a wide variety of routinely collected local National Health Service (NHS) data sources. No new data was collected, and no single data source was available, which provided a fully informed perspective; therefore, a purposive method was chosen. The following data sources were included and were sub-analysed by measures of equity including age, sex, ethnicity, and postcode wherever possible:

(a) Electronic patient record (EPR) data: The local community and secondary care eye hospitals use an administration system supplied by Medisoft Limited, ${ }^{7}$ which is linked to the wider patient administration system (PAS). A subset of their dataset from 2002 to 2007 inclusive was audited, patients were included in the analysis if they lived within Leeds primary care trust (PCT) boundaries, and a date of diagnosis was available. A diagnosis in both eyes of any of the following was required: POAG; normal pressure glaucoma; or chronic open angle glaucoma. Patients with secondary glaucoma, suspected glaucoma, and other forms of glaucoma were excluded. The first clinic location, age at diagnosis, sex, ethnicity, and last recorded postcode were analysed. Visual field information had been imported into the Medisoft database, and analysis was possible using electronic PAS data to provide postcode data. Mean deviation (MD) of visual field at presentation was used as a marker of lateness of presentation as it has been shown to have a linear relationship with health-related quality of life. ${ }^{8}$ A threshold of MD at presentation in the worst affected eye of $<-12 \mathrm{~dB}$ was used as a marker of late presentation. ${ }^{9}$ Further analysis was conducted on the stage at presentation and patient characteristics.

(b) Primary care prescribing data: The electronic prescribing analysis and cost tool was used, which covers prescriptions prescribed in primary care in England, was reviewed for the 3 financial years 2005/2006 to $2007 / 2008$.

(c) Certifications for visual impairment (CVI): CVI's from the local secondary care provider were available for the 
3 years from January 2005 to December 2007 inclusive. The dataset was cleaned for duplicates and patients registered with other PCTs.

(d) Hospital episode statistics data: Hospital episode statistic data was reviewed as follows:

- Diagnostic coding is generally not available for outpatient appointments, so outpatient data was extracted from PAS for all ophthalmology first attendances (2000-2006) and for the two specific ophthalmic consultants sub-specialising in glaucoma (2002/3-2007/8).

- Inpatient admissions are rare for POAG, but are coded with ICD-10 codes H40.0-42.8, these were reviewed from $2002 / 2003$ to $2007 / 2008$. Surgical procedures are also relatively uncommon, but are coded by procedure (OPCS) and data was, therefore, available for analysis for procedures conducted from 2002/2003 to 2007/ 2008 for C60.1 trabeculectomy, C60.5 insertion of drainage tube, and C61.1 laser trabeculectomy.

(e) Locally collected general practitioner with special interest (GPwSI) audit data: There were two GPwSIs in ophthalmology who are delivering community-based eye care in Leeds. Their audit data was reviewed. (f) GOS routinely collected data available from the NHS Information Centre.

(g) A local qualitative study of barriers to the uptake of eye care services in the older African-Caribbean population in Leeds has been earlier reported in brief $f^{10}$ and was used to understand local barriers to access in the equity profile. A recent internal evaluation of community eye services using semi-structured interviews, questionnaire, and focus groups was also referred to.

The final step in the equity profile was to make recommendations for action.

Statistical analysis was conducted using Microsoft Excel 2003. Ethical approval was not required.

\section{Results}

\section{Mapping of Leeds Eye Care Services}

In addition to standard general practice, there are also two GPwSIs providing ophthalmic care in their practices. Optometrist referral is the principal route by which patients with glaucoma are picked up across the city. Figure 1 shows the distribution of opticians premises

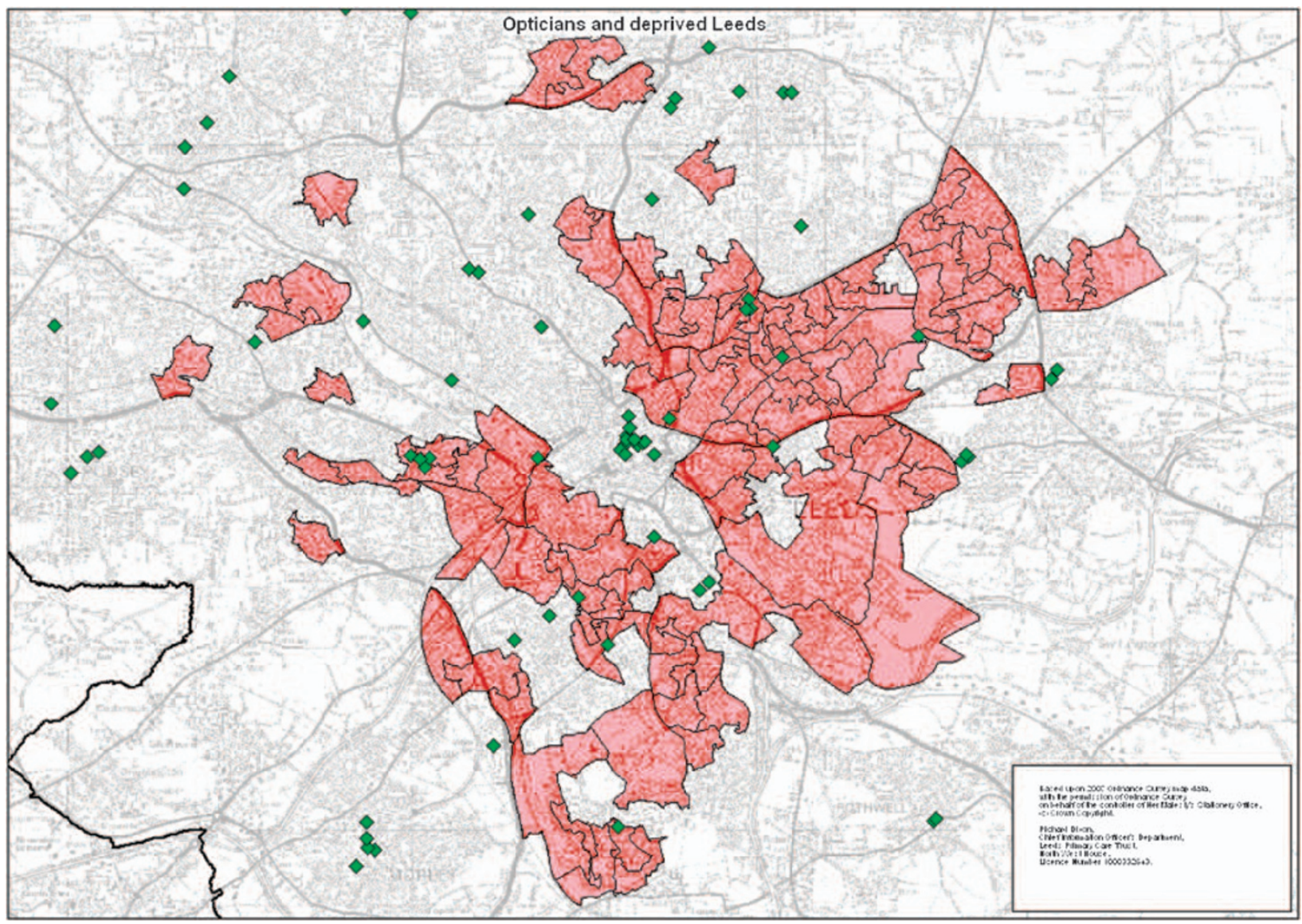

Figure 1 Location of opticians compared with areas of high relative deprivation in Leeds. 
in Leeds (denoted by green diamonds), with the areas of the city, which are included in the $10 \%$ nationally most deprived LSOA (highlighted in red), these areas are both more densely populated and have higher proportions of people of Black ethnic origin than the non-highlighted more affluent areas. A clear mismatch between the most deprived LSOA and the location of opticians premises is clear.

Two integrated primary/secondary care centres exist in the most deprived areas of Leeds. Leeds Teaching Hospitals NHS Trust (LTHT) provides the majority of the ophthalmology care at St James's University Hospital, located in an area of high deprivation, and also staffs outpatient services at a district general hospital in a geographically distant, but affluent area of Leeds.

\section{Assessment of need}

\section{Glaucoma}

A crude estimate of between 5963 and 6700 people currently with chronic open angle glaucoma in
Leeds was produced. It is accepted that it is a crude calculation, which does not include glaucoma suspects, patients with ocular hypertension, nor false-positive subjects who are referred. Not all of these subjects with glaucoma will present to health services. Figure 2 shows the estimated numbers of people with glaucoma by general practice. The shaded grey area refers to the PCT boundary. The red circles are located at the sites of general practices, the bigger the circle, the larger the estimated glaucoma population. Presenting data in this way also helps to identify where future community-based eye services might usefully be located.

\section{Age and ethnicity}

Glaucoma prevalence rises with increasing age, its prevalence in a Caucasian population is estimated at $1-2 \%$ of those over the age of 40 years, rising to $10 \%$ over the age of 75 years. $^{2}$ It is approximately four times more common, develops at an earlier age, and follows a more aggressive clinical course in individuals of African-Caribbean descent. ${ }^{11,12}$ The relative prevalence

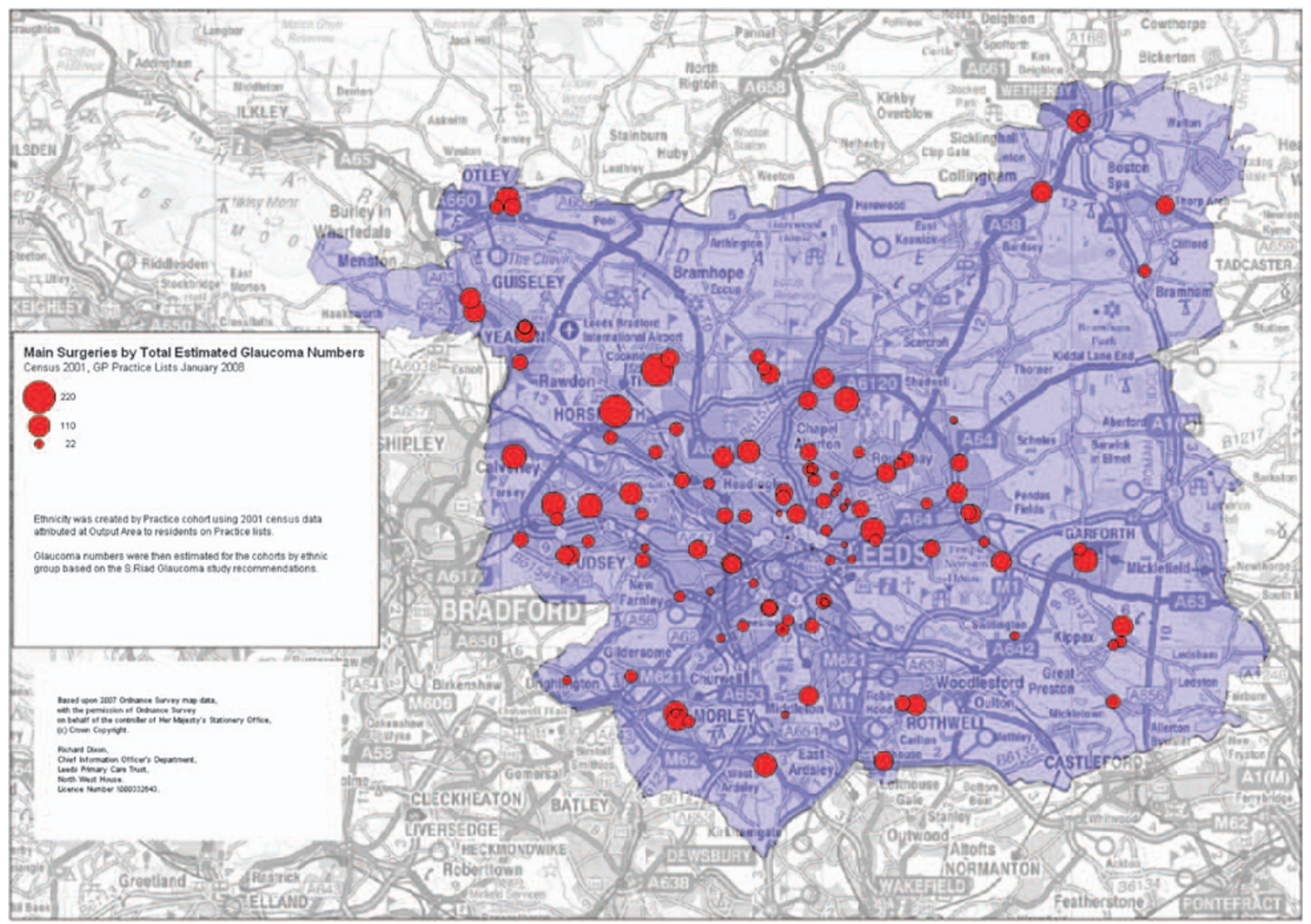

Figure 2 Estimated numbers by practice. 
Table 1 EPR (Medisoft) data on glaucoma patients on stage of presentation $(n=1275)$

\begin{tabular}{|c|c|c|c|c|c|c|c|}
\hline $\begin{array}{l}\text { Mean field in one or more } \\
\text { eye }(d B)\end{array}$ & $\begin{array}{l}\text { Males } \\
\mathrm{n}(\%)\end{array}$ & $\begin{array}{c}\text { Females } \\
\mathrm{n}(\%)\end{array}$ & $\begin{array}{l}\text { Total (inc gender } \\
\text { unknown) }\end{array}$ & $\begin{array}{l}\text { Mean age in } \\
\text { years }(95 \% \mathrm{CI})\end{array}$ & $\begin{array}{l}\text { Age range } \\
\text { (years) }\end{array}$ & $\begin{array}{c}\text { Median } \\
\text { (years) }\end{array}$ & $\begin{array}{c}\text { Percentage of all patients } \\
\text { with field recorded }\end{array}$ \\
\hline $\begin{array}{l}\text { Neither eye has score of } \\
<-12 \text { ('early presentation') }\end{array}$ & $425(72.0)$ & $472(70.3)$ & 908 & 72.38 (71.67-73.09) & 27-97 & 74 & 71.0 \\
\hline $\begin{array}{l}\text { One or both eyes have score } \\
\text { of }<-12 \text { ('late presentation') }\end{array}$ & $165(28.0)$ & 199 (29.7) & 367 & 76.35 (75.13-77.57) & $14-98$ & 78 & 29.0 \\
\hline
\end{tabular}

of the over 65 years population and the Black ethnic population was presented in the Equity Profile using GIS mapping.

\section{Family history}

No information source was available to permit mapping the distribution of close relatives of patients diagnosed with glaucoma.

\section{Leeds and deprivation}

A total of 150788 people in Leeds live in the most deprived $10 \%$ of communities (LSOA) in England (Figure 1). These tend to comprise both younger populations and higher ethnic minority populations. Leeds more deprived SOA have a slightly younger age profile and contain over 2.5 times more people from Black or Black British ethnic backgrounds. ${ }^{13}$

\section{Analysis of data sources for equity}

\section{EPR data}

Data was extracted for 1771 Leeds PCT patients from 2002 to 2007 inclusive. Patients with secondary glaucoma, suspected glaucoma, and other forms of glaucoma were excluded leaving 1358 patients with glaucoma in the analysis. Ethnicity status was poorly recorded as it was only available through the PAS system: only $9 \%$ of outpatient attendances had ethnicity recorded (see below). The anticipated clustering in areas of high population density and areas with high proportion of older or African-Caribbean residents was not observed, suggesting that relatively fewer cases were being detected and referred from these areas. This possibility was investigated statistically by correlating whether the patient lived within an area of high multiple deprivation to MD of visual field at presentation, as a surrogate for late presentation. Data on fields at presentation was available for 1275 glaucoma patients. A total of 367 (28.8\%) of those for whom fields were available were found to have presented 'late' (Table 1).

Of the 367 glaucoma patients who presented 'late', 95 had an MD score of $<-18 \mathrm{~dB}$, with the lowest recorded $\mathrm{MD}$ in either eye being $-30 \mathrm{~dB}$.

Analysis of stage at presentation and patient characteristics found a statistically significant difference in age and stage of presentation. The late presenters were more likely to be significantly older (mean age $=76.4$ years, 95\% CI $=75.1-77.6)$ compared with the earlier presenters (72.4 years, 95\% CI $=71.7-73.1$ ). This is an important finding and is in keeping with the published literature, showing that older people are accessing services at a later stage of disease presentation.

Analysis of postcode data and stage at presentation showed that patients living in more deprived areas were more likely to present late. Of the population living in the most deprived 10\% LSOAs, 169 (64.5\%) presented early and $92(35.5 \%)$ presented late. Of those not living in the most deprived 10\% LSOAs, 731 (72.9\%) presented early and $272(27.1 \%)$ presented late. This difference was statistically significant $\left(\chi^{2}=7.1,1 \mathrm{df}, P<0.05\right)$. This is also an important finding, in keeping with the published literature, and is a marker of access to eye tests and/or eye care services, showing that people living in deprived areas are accessing services at a later stage of disease presentation. This may be directly related to the location of primary eye care services as shown in Figure 1.

No significant difference in the stage at presentation was found between men and women $\left(\chi^{2}=0.44,1 \mathrm{df}\right.$, $P>0.05)$. Owing to the small numbers of patients with recorded ethnicity status, stage of presentation by ethnicity could not be analysed.

Mapping of absolute numbers of patients and the proportion of patients presenting late divided by 2004 electoral wards across Leeds was conducted. Areas with fewer than expected patients, or higher proportions of patients presenting late, could, therefore, be targeted for further study.

\section{Primary care prescribing data}

A total of 220988 items of glaucoma-specific ocular drugs were dispensed in primary care during this time, at an average cost of $£ 1.1 \mathrm{M}$ per year. Prescriptions for glaucoma can be broken down and analysed by GP practice. Although age and gender weighting was possible, it was not possible to weight data for other relevant demographics such as deprivation or ethnicity. Variation between practices was evident across the city, but no robust analysis was possible to allow determination whether there was evidence of inequity. 
Hospital episode statistics data

In general, the hospital episode statistics data was of insufficient quality to allow robust equity analysis. Only $9 \%$ of outpatient appointments were coded for ethnicity, although this was better for inpatients (45\%); however, the number of inpatients was small (298/32549 ophthalmic procedures conducted over the 5-year period were for glaucoma-specific procedures), hence no meaningful sub-analysis was possible.

\section{Certifications for visual impairment}

A total of 1097 Leeds PCT patients had been registered from LTHT as sight impaired or severely sight impaired over the 3 years, of whom 1060 had a cause recorded. Of those with a cause recorded, 106 (10\%) had POAG recorded as being a reason for their sight loss, and 69 of these had POAG recorded as the sole or the primary cause. Of the 106 patients who lost their sight for a reason including POAG, the mean age at registration for males $(n=40)$ and females $(n=66)$ was similar being 79.0 years (95\% CI $=74.8-83.2)$ and 78.9 years $(95 \% \mathrm{CI}=75.0-82.8)$, respectively. No ethnicity data was available.

\section{General practitioner with special interest}

There are two GPwSIs in ophthalmology delivering community-based eye care in Leeds. Although routine data collection in primary care is often superior to that in secondary care, and one GPwSI had seen 218 patients regarding glaucoma in 2007-2008, it was not possible to undertake meaningful analysis to inform the equity profile.

\section{GOS data}

This showed that the rates of NHS sight testing were lower in Leeds than neighbouring PCTs, the regional average, and the national average. Lower than average proportions of children, people on low incomes, and glaucoma/diabetics were receiving NHS sight tests. A slightly increased prevalence of testing in relatives of a glaucoma sufferer and over the age of 40 was found.

\section{Local qualitative data}

Two local qualitative studies have been undertaken in Leeds and were used to inform the glaucoma equity profile. Relevant findings from a qualitative study into barriers to access for the African-Caribbean population included lack of awareness of risk; fatalist beliefs about glaucoma; costs of eye tests; distance to optometry and associated transport difficulties; traditional folk practices; and poor communication. In the internal service evaluation of community eye care, service users had returned 211/408 questionnaires and 88\% reported a journey time of $<30 \mathrm{~min}$ with $85 \%$ finding the location more convenient than central hospitals. Comments from interview and focus groups were almost exclusively positive. Such data is useful in the creation of an equity profile, as it provides encouragement to proceed with further decentralisation in which location issues are felt to be contributory to any identified inequity.

\section{Recommendations}

Recommendations were made to the PCT regarding the need for future action to reduce inequalities in glaucoma detection and care.

\section{Discussion}

Conducting the glaucoma equity profile has provided both the local health care commissioners and providers with a picture of the extent of the under-detection and under-provision of glaucoma care to those in areas of multiple deprivation in the city, some of whom are at increased risk because of their ethnicity. This study shows that the way that services are currently constructed in our population has failed to promote equity in access for those with the greatest need.

\section{Limitations}

This study was not a formal epidemiological survey, but painted a picture using routinely collected data sets, each with different potential problems and biases, to triangulate the data. The quality of the routine data was variable. Although our primary focus was on chronic glaucoma, where necessary, we used data which contained a mixture of glaucoma diagnoses. The data from Medisoft potentially provides a rich source of information, but needs to be interpreted with a degree of caution relating to the degree of coverage of the whole new glaucoma population in contact with services. It is not thought that there is any obvious reason for selection bias of patients who are recorded on Medisoft, which gives greater confidence in the results. The poor quality of ethnicity data recording was disappointing, especially given the strong ethnic risk in this disease. National initiatives to improve ethnicity data recording are now in place. The equity profile needs to be repeated in a few years to assess the impact of the recommendations on patient outcomes and healthcare processes.

\section{Strengths of the equity profile}

Although direct research methodologies such as population-based surveys or case-control studies of late presenters would answer questions regarding equitable access, the advantage of the equity profile design is that it 
uses routinely collected data. It is easily repeatable in a cycle of equity audit and does not require the additional funding, logistical, and technical expertise or resources mandatory in conduction of such research projects. Although many of the avenues of investigation provided no useful information, we present each exploration to show the range of sources that should be investigated in such a process as this. We were fortunate to have a well-established EPR system in Leeds, which provided the strongest evidence, free from obvious source of bias, that living in an area of deprivation was predictive of later presentation, and by inference that there was inequity in access to glaucoma services.

Using the direction provided by the equity profile, any service restructuring or move to increase service provision capacity in the city can be designed to target the communities, which are under-represented in the current workload of the hospital eye service, and, therefore, presumed to have higher rates of undiagnosed disease. We have since targeted one area of the city, with a high proportion of residents being of African-Caribbean origin, and hence more likely to be at risk of glaucoma. This area is not currently served by a local optometric practice. We aim to increase the early detection of glaucoma, through various health promotion campaigns. These included an 'eye stand' at the local Carnival, and refraction and ocular health checks in the local Community Centres. This work is currently being evaluated.

Increasing access to services involves collaboration with optometrists, ophthalmologists, public health, and those commissioners who are ultimately responsible and accountable for the health of the local population. It is no longer acceptable to rely on private high street optometry to provide primary eye care services in areas of high need. Outreach services, which provide free sight tests and a 'no cost barrier' service must be developed and evaluated in areas of relative deprivation.

\section{Conclusion}

As a cause of inequalities, sight loss from glaucoma leads to poverty, loss of independence and social exclusion, and leads to comorbidities including accidents, falls, and generally reduced health-related quality of life measures. ${ }^{14,15}$ The cost of leaving a section of society underserved is borne by those individuals and families least able to afford that cost. The opportunity exists for the ophthalmologist to take a pro-active lead in addressing the inequity of access to services. Such initiatives might start with producing an equity profile, a process that could be readily repeated for other ophthalmic conditions such as cataract and age-related macular degeneration.

\section{Summary}

What was known before

- Glaucoma is a cause and a consequence of health inequalities. The equity profile is an established public health tool to measure the extent of health inequalities in a population and is used to inform the commissioning cycle to achieve more equitable health.

What this study adds

- This is the first time that an equity profile has been conducted for an ophthalmic condition. The methodology can be replicated for any population and most ophthalmic conditions. Private high street optometry cannot be relied on to find cases of glaucoma in high-risk populations. We found a mismatch between location of optometry and areas of high deprivation in the city of Leeds. Inequalities in glaucoma-risk factors, access to services exist, and must be systematically addressed by local commissioners if World Class commissioning and World Class Eye services are to be achieved.

\section{Conflict of interest}

The authors declare no conflict of interest.

\section{Acknowledgements}

We thank Ben Woodhouse, Medicines Economist and Locality Lead Pharmacist, NHS Leeds; Kath Lengle, CVI Database Manager, Leeds Teaching Hospitals NHS Trust; Medisoft Limited, Leeds Innovation Centre, 103 Clarendon Road, Leeds, LS2 9DF; Kay Hollingworth, Ophthalmic Public Health Fellow, NHS Leeds and Leeds Teaching Hospitals NHS Trust for supporting this work.

\section{References}

1 Royal College of Opthalmologists. Guidelines for the Management of Open Angle Glaucoma and Ocular Hypertension. RCO: London, 2004.

2 National Institute for Health and Clinical Excellence (NICE). CG85: Diagnosis and Clinical Management of Chronic Open Angle Glaucoma and Ocular Hypertension. NICE: April 2009 http:/ / guidance.nice.org.uk/CG85/NiceGuidance/ pdf/English. (accessed 4/11/09).

3 Burr JM, Mowatt G, Hernandez R, Siddiqui MA, Cook J, Lourenco $\mathrm{T}$ et al. The clinical effectiveness and costeffectiveness of screening for open angle glaucoma: a systematic review and economic evaluation. Health Technol Assess 2007; 11(41): iii-iv, ix-x, 1-190.

4 Fraser S, Bunce C, Wormald R, Brunner E. Deprivation and late presentation of glaucoma: case-control study. BMJ 2001; 322(7287): 639-643.

5 Health Development Agency. Health Equity Audit Made Simple: A briefing for Primary Care Trusts and Local Strategic Partnerships. Working document January 2003. http:/ / www.nice.org.uk/aboutnice/whoweare/aboutthehda/ hdapublications/health_equity_audit_made_simple.jsp. (accessed 4/11/09). 
6 Riad S. Report to the Royal College of Ophthalmologists Professional Standards Committee: the Primary Care of Chronic Open Angle Glaucoma. RCO: London, 2008.

7 Medisoft Limited. Leeds Innovation Centre, 103 Clarendon Road, Leeds, LS2 9DF, UK.

8 McKean-Cowdin R, Varma R, Wu J, Hays RD, Azen SP, Los Angeles Latino Eye Study Group. Severity of visual field loss and health-related quality of life. Am J Ophthalmol 2007; 143(6): 1013-1023.

9 Kanski JJ. Clinical Ophthalmology, 6th ed. Butterworth Heinemann Elsevier: Philadelphia, USA, 2007, p 387.

10 Awobem JF, Cassels-Brown A, Buchan JC, Hughes KA. Exploring glaucoma awareness and the utilization of primary eye care services: community perceived barriers among elderly African Caribbeans in Chapeltown, Leeds. Eye 2009; 23(1): 243.
11 Poinoosawmy D, Nagasubramanian S, Wormald R, Hitchings R. Glaucoma and race. Lancet 1989; 1(8647): 1134

12 Munoz B, West SK. Blindness and visual impairment in the Americas and the Caribbean. Br J Ophthalmol 2002; 86(5): 498-504.

13 Leeds Joint Public Health Information Group. Measuring the Gap: Tackling Health Inequalities in Leeds 2007. http:/ / www.leedspct.nhs.uk/.

14 Freeman EE, Munoz B, Rubin G, West SK. Visual field loss increases the risk of falls in older adults: the Salisbury eye evaluation. Invest Ophthalmol Vis Sci 2007; 48(10): 4445-4450.

15 Haymes SA, Leblanc RP, Nicolela MT, Chiasson LA, Chauhan BC. Risk of falls and motor vehicle collisions in glaucoma. Invest Ophthalmol Vis Sci 2007; 48(3): $1149-1155$. 